\title{
Clinical Characteristics of Sphenoid Sinus Fungal Ball Patients With Visual Disturbance
}

\author{
Jong Sei Kim • Byung Kil Kim • Sang Duk Hong ${ }^{1}$ Hyung Jin Kim² • Hyo Yeol Kim \\ Departments of ${ }^{l}$ Otorhinolaryngology-Head and Neck Surgery, ${ }^{2}$ Radiology, Samsung Medical Center, \\ Sungkyunkwan University School of Medicine, Seoul, Korea
}

Objectives. A sphenoid sinus fungal ball is a rare disease that can cause visual disturbances. Most afflicted patients remain in an indolent state. However, once the visual disturbance has occurred, the recovery rate is very low. The purpose of this study was to overview the clinical characteristics of patients with a sphenoid sinus fungal ball and ascertain factors possibly related to the occurrence of a visual disturbance.

Methods. We retrospectively reviewed the medical records of all patients who underwent endoscopic sinus surgery for a sphenoid sinus fungal ball at our hospital. We enrolled 47 patients in this study.

Results. Old age and a female predominance were noted. Nasal symptoms were the most common symptom. Eight patients showed visual disturbances. We also compared the clinical characteristics between patients with and without visual disturbances in univariate analysis. Old age, underlying diabetes mellitus, and a sphenoid sinus wall defect visible by computed tomography were factors significantly related to the occurrence of visual disturbances. But only sphenoid sinus wall defect showed significance in multivariate analysis.

Conclusion. It is important to prevent complications, such as visual disturbance, in patients with a sphenoid sinus fungal ball. This study describes an early surgical treatment that may be required for patients with a sphenoid sinus fungal ball and particularly patients with a sphenoid sinus wall defect.

Keywords. Sphenoid Sinus; Aspergillosis; Vision Disorders

\section{INTRODUCTION}

A fungal ball (FB) in the paranasal sinus is an extramucosal mycotic proliferation that fills one or more paranasal sinuses [1]. The most frequently involved is the maxillary sinus followed by the sphenoid sinus. The prevalence of fungus balls is increasing rapidly in older age patients and those who have undergone endodontic treatment, particularly in the maxillary sinuses [2].

However, the diagnosis of a sphenoid sinus FB is often de-

\footnotetext{
- Received October 16, 2015

Revised December 17, 2015

Accepted December 22, 2015

- Corresponding author: Hyo Yeol Kim

Department of Otorhinolaryngology-Head and Neck Surgery, Samsung

Medical Center, Sungkyunkwan University School of Medicine,

81 Irwon-ro, Gangnam-gu, Seoul 06351, Korea

Tel: +82-2-3410-3579, Fax: +82-2-3410-3879

E-mail: siamkhy@skku.edu
}

layed, particularly in patients with headache that may persist for several years before the disease is diagnosed [3]. Because symptoms are non-specific, the possibility of the disease is often overlooked, and most patients are not diagnosed with sphenoid FB until complications occur or by chance at a regular health checkup.

Involvement of a FB in the sphenoid sinus comprises $4.5 \%$ to $26.8 \%$ of cases with FB [4]. The size and pneumatization of the sphenoid sinus varies and many important structures lie adjacent to the sphenoid sinus (e.g., cavernous sinus, pituitary gland, internal carotid artery, and cranial nerves II, III, IV, V1, V2, and VI) [5]. Because of these structural characteristics, sphenoid sinusitis can cause serious complications, including headache and visual disturbances; however, few studies have focused on the relationship between a sphenoid sinus FB and orbital complications and possible factor associated with an orbital disturbance.

The focus of this study is to report the clinical features of

Copyright $@ 2016$ by Korean Society of Otorhinolaryngology-Head and Neck Surgery

This is an open-access article distributed under the terms of the Creative Commons Attribution Non-Commercial License (http://creativecommons.org/licenses/by-nc/4.0)

which permits unrestricted non-commercial use, distribution, and reproduction in any medium, provided the original work is properly cited. 
sphenoid sinus FB with or without ophthalmic complications. Furthermore, we tried to determine the factors related to the occurrence of visual disturbance so physicians can perform earlier surgical interventions in those patients before visual disturbances occur.

\section{MATERIALS AND METHODS}

This retrospective cohort study was conducted in the department of otolaryngology of Samsung Medical Center between January 1997 and April 2013. The study was approved by the Institutional Review Board of Samsung Medical Center (201409-098-003). We reviewed the medical records of patients with sphenoid sinus FB. The inclusion criteria were: (1) involvement of the sphenoid sinus, as detected by computed tomography (CT); and (2) a pathologically confirmed fungal aggregate in the sphenoid sinus. Patients were excluded from this study if they had other sphenoid sinus diseases (e.g., mucocele, lymphoma, or malignancy). Patients with other kinds of fungal sinusitis were also excluded, including those with invasive fungal sinusitis with mucosal invasion and necrosis or allergic fungal sinusitis revealing allergic mucin. Surgery was usually done endoscopically through the endonasal paraseptal or transethmoidal route under general anesthesia, and a biopsy of the fungal aggregate was taken during the surgical procedures. The surrounding sphenoid mucosa was also biopsied when the patient had a visual disturbance or severe headache.

Demographic data, presence of nasal symptoms, headache, underlying diabetes mellitus (DM), and radiological findings were reviewed. In addition, an antifungal medication, treatment outcome, and the time interval between symptom occurrence and surgery were also reviewed in patients with visual disturbances. Characteristic CT findings were reviewed to identify intrasinus opacification, a defect and sclerosis of the sphenoid sinus bony wall, and other sinus lesions [6,7]. Magnetic resonance imaging (MRI) was performed preoperatively in patients with visual disturbances. The patients were divided into 2 groups based on the presence of a visual disturbance. We compared the characteristics of the 2 groups to reveal factors associated with the occurrence of a visual disturbance. The following variables

\section{H I}

- Risk factors of visual disturbance were investigated in 47 patients with sphenoid sinus fungal ball.

- Visual disturbance occurred in 18 of 47 (17\%) patients.

- Old aged, diabetic, and CT-sphenoid bony defect-containing patients were more likely to have visual disturbance.

- However, the risk factors were not confirmed by the multivariate analyses. were analyzed to determine the factors associated with a visual disturbances patients with a sphenoid FB: bony defect or sclerosis of sphenoid sinus wall, fullness of sphenoid sinus opacification on CT images, presence of nasal symptoms, including purulent rhinorrhea, postnasal drip, and headache, and the presence of underlying DM. Involvement of the optic nerve and cavernous sinus as detected by MRI was evaluated in patients with visual disturbances.

The two sample $t$-test was performed to compare mean ages of the 2 groups. Fisher exact test was used to compare differences in the associated clinical manifestations between the patients with a FB with and without a visual disturbance. Logistic regression analysis using Firth's penalized maximum likelihood estimation method was conducted to investigate the clinical manifestations associated with visual disturbance due to a rare event. Clinical manifestations with $P$-values $<0.05$ in the univariate analysis were included in the logistic regression analysis. The data were analyzed using SAS ver. 9.3 (SAS Institute Inc., Cary, NC, USA). A $P$-value $<0.05$ was considered significant.

\section{RESULTS}

A total of 425 patients were diagnosed with FB in the paranasal sinuses from January 1997 to April 2013, and 47 of them (11\%) had a sphenoid sinus FB. The enrolled patients consisted of 11 men and 36 women (1:3.3) with a mean age of 59.8 years (range, 36 to 85 years). Eleven patients $(23 \%$ ) were diabetic but no other immunodeficient patients were included. All patients underwent endoscopic nasal examinations at the first visit and a $\mathrm{CT}$ scan was obtained. MRI was performed on the first visit in 8 patients who had visual disturbances. The mean follow-up period was $13.6 \pm 20.3$ months (range, 0.1 to 104.6 months; median, 4.8 months).

Nasal symptoms were the most common symptom at the first visit. Twenty-seven patients presented with nasal symptoms, including purulent rhinorrhea (11, 41\%), nasal obstruction (8, $30 \%)$, post nasal drip $(19,70 \%)$, and hyposmia $(4,15 \%)$. Twenty-six patients suffered from headache (frontal, retro-orbital, temporal, or occiput), which was the second most common symptom. Eight patients had visual disturbances, including extraocular muscle restriction or impaired visual acuity and underwent an immediate endoscopic sphenoidotomy. The surgery was usually done through the sphenoethmoidal recess. Fungal debris was removed with a large sphenoidotomy and the areas was irrigated profusely remove all fungal debris, which was followed by a mucosal biopsy. After the surgery, an antifungal agent (amphotericin B followed by voriconazole) and systemic steroid (dexamethasone) were administered to the patients with visual disturbances. Although the biopsy results were inconsistent with invasive fungal sinusitis, we decided to use systemic antifungal agents in all cases under consultation with the infec- 
tious disease department because of cranial nerve involvement.

All radiographic studies revealed space-occupying lesions in the sphenoid sinus, and 27 patients (57\%) had complete opacity of both affected and unaffected sphenoid sinuses; only one patient had bilateral partial opacity in sphenoid sinuses. Otherwise, the remaining 19 patients (40\%) had unilateral complete opacity of the affected sphenoid sinus. Microcalcification was detected in the sphenoid sinuses of all patients. Defects in the sphenoid sinus bony wall were observed in 19 patients $(40 \%)$,
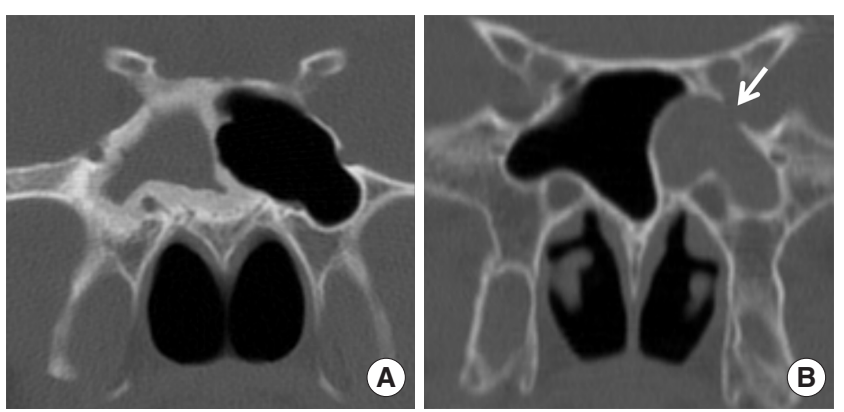

Fig. 1. Computed tomography scans of different presentations of a sphenoid sinus fungal ball. Both images show total opacity of the sphenoid sinuses. (A) Sclerosis of the sphenoid sinus bony wall. (B) Sphenoid sinus bony wall defect (arrow).
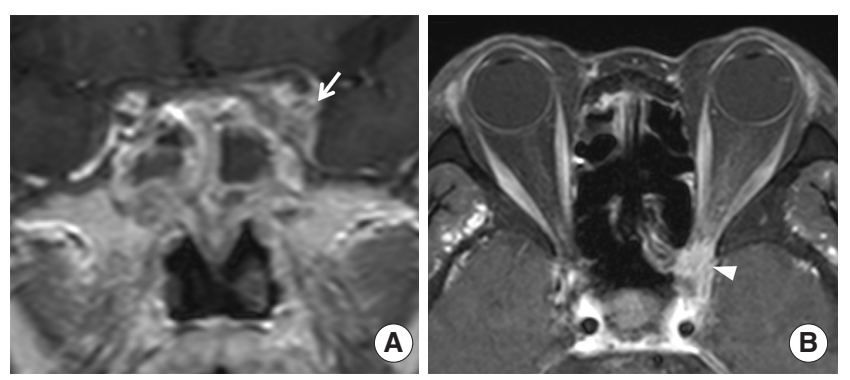

Fig. 2. Magnetic resonance imaging (MRI) presentation of a sphenoid sinus fungal ball in patient with a visual disturbance. (A) T1weighted coronal image showing intermediate signal intensity filling of the sphenoid cavity and enhanced lesions of the cavernous sinus (arrow). (B) T1-weighted axial image showing orbital apex (arrowhead) on an orbital MRI. and sclerosis of the sphenoid sinus bony wall was observed in 30 patients $(64 \%)$ (Fig. 1). The clinical and radiological characteristics of these patients are summarized in Table 1 . No recurrence of a sphenoid sinus FB occurred after endoscopic surgery.

We compared the clinical characteristics of the patients with or without visual disturbances in univariate analysis (Table 2). A visual disturbance was observed in 8 patients $(17 \%)$, and 5 of these 8 patients with a visual disturbance also presented with an extraocular muscle restriction on the ophthalmologic examination. MRI was performed in these patients, and all patients had enhancing lesions involving the optic nerve or cavernous sinus on T1-weighted gadolinium MRI images (Fig. 2). A visual disturbance accompanied the FB more frequently in the elderly $(P=0.033)$. Six patients $(75 \%)$ with visual disturbance had DM $(P=0.001)$ compared with $13 \%$ of the patients without a visual disturbance. The average of fasting blood glucose level was $185.5 \pm 47.1$ in the DM patients. No significant difference in symptomatology was observed between the groups. All patients

Table 1. Clinical characteristics of the study patients with a sphenoid sinus fungal ball $(n=47)$

\begin{tabular}{lr}
\hline Characteristic & Value \\
\hline Sex & \\
Men & $11(23)$ \\
Women & $36(77)$ \\
Age (yr) & $59.7 \pm 13$ \\
Diabetes mellitus & $11(23)$ \\
Nasal symptom & $25(53)$ \\
Headache & $21(45)$ \\
Extraocular muscle restriction & $5(11)$ \\
Visual loss & $8(17)$ \\
Computed tomography findings & \\
Wall defect & $19(40)$ \\
Wall sclerosis & $30(64)$ \\
Bilateral complete opacity & $27(57)$ \\
Bilateral partial opacity & $1(2)$ \\
Unilateral complete opacity & $19(40)$ \\
\hline
\end{tabular}

Values are presented as number (\%) or mean \pm SD.

Table 2. Comparison of the clinical characteristics between the 2 groups with univariate analysis and multivariate analysis

\begin{tabular}{|c|c|c|c|c|c|}
\hline Variable & With VD $(n=8)$ & Without VD $(n=39)$ & $P$-value ${ }^{a)}$ & $P$-value ${ }^{b)}$ & OR $(95 \% \mathrm{CI})^{\mathrm{b})}$ \\
\hline Age (yr) & $67.5 \pm 10.57$ & $58.21 \pm 10.57$ & $0.033^{\star}$ & 0.220 & $1.060(0.966-1.164)$ \\
\hline Sex (male:female) & $3: 5$ & $8: 31$ & 0.367 & & \\
\hline Diabetes mellitus & $6(75)$ & $5(13)$ & $0.001^{*}$ & 0.093 & $5.391(0.756-38.451)$ \\
\hline Headache & $7(87)$ & $19(48)$ & 0.059 & & \\
\hline Nasal symptom & $2(25)$ & $25(64)$ & 0.057 & & \\
\hline Defect $^{c)}$ & $8(100)$ & $11(28)$ & $<0.001^{*}$ & $0.046^{*}$ & $20.168(1.044-389.619)$ \\
\hline Opacity ${ }^{d)}$ & $4(50)$ & $23(59)$ & 0.707 & & \\
\hline Sclerosis ${ }^{e)}$ & $4(50)$ & $26(67)$ & 0.435 & & \\
\hline
\end{tabular}

Values are presented as mean \pm SD or number (\%).

VD, visual disturbance; OR, odds ratio; $\mathrm{Cl}$, confidence interval.

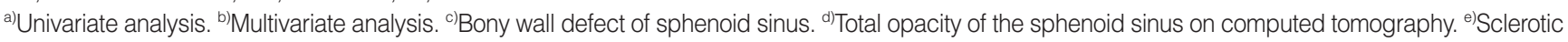
change in the sphenoid sinus bony wall. ${ }^{*} P<0.05$. 
Table 3. Clinical and radiological presentations of the patients with a visual disturbance

\begin{tabular}{|c|c|c|c|c|c|c|c|c|c|c|c|}
\hline \multirow{2}{*}{ Patient } & \multirow{2}{*}{$\begin{array}{l}\text { Age } \\
(y r)\end{array}$} & \multirow{2}{*}{$\begin{array}{l}\text { Durationa) } \\
\text { (day) }\end{array}$} & \multicolumn{2}{|c|}{ Extraocular muscle restriction } & \multicolumn{2}{|c|}{ Visual acuityb) } & \multirow{2}{*}{$\begin{array}{l}\text { Diabetes } \\
\text { mellitus }\end{array}$} & \multirow{2}{*}{ Headache } & \multirow{2}{*}{ Defect ${ }^{c)}$} & \multirow{2}{*}{ Sclerosis ${ }^{\mathrm{d})}$} & \multirow{2}{*}{ Opacity } \\
\hline & & & Preop & Postop & Preop & Postop & & & & & \\
\hline 1 & 60 & 10 & + & + & $\mathrm{LP}(-)$ & $\mathrm{LP}(-)$ & - & + & + & - & + \\
\hline 2 & 47 & 98 & + & + & $20 / 200$ & $\operatorname{LP}(-)$ & + & + & + & - & - \\
\hline 3 & 85 & 14 & + & + & $\mathrm{LP}(-)$ & $\mathrm{LP}(-)$ & - & - & + & - & + \\
\hline 4 & 80 & 33 & + & - & $\mathrm{LP}(+)$ & $\mathrm{FC}^{f)}(10)$ & + & + & + & + & - \\
\hline 5 & 62 & 115 & + & - & $20 / 50$ & 20/22 & + & + & + & + & + \\
\hline 6 & 68 & 11 & - & & $20 / 200$ & $20 / 20$ & + & + & + & - & + \\
\hline 7 & 77 & 56 & - & & $\operatorname{LP}(-)$ & $\mathrm{LP}(-)$ & + & + & + & + & - \\
\hline 8 & 61 & 3 & - & & $\operatorname{LP}(+)$ & $\operatorname{LP}(-)$ & + & - & + & + & - \\
\hline
\end{tabular}

Preop, preoperative; Postop, postoperative; LP, light perception.

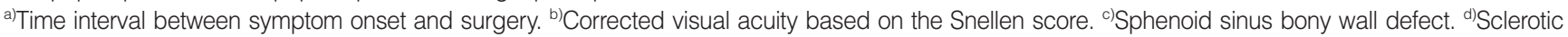
change in the sphenoid sinus bony wall. ${ }^{\text {e}}$ Bilateral complete opacity of the sphenoid sinus on computed tomography. ${ }^{\mathrm{f}}$ Finger count and distance (cm).

with visual disturbances and $28 \%$ of patients without a visual disturbance had a bony wall defect detected by the radiological examination $(P<0.001)$. No differences were observed for the presence of complete opacity or sclerosis of the sphenoid sinus bony wall.

A multivariate analysis was conducted, including a bony defect of the sphenoid sinus, DM, and age as independent variables to adjust for their confounding effects, and a defect in the sphenoid sinus bony wall was significantly associated with the presence of a visual disturbance $(P=0.046$; odds ratio [OR], 20.168; 95\% confidence interval [CI], 1.044 to 389.619). Age $(P=0.220 ;$ OR, $1.06 ; 95 \% \mathrm{CI}, 0.966$ to 1.164$)$ and $\mathrm{DM}$ ( $P=0.093$; OR, 5.391; 95\% CI, 0.756 to 38.451$)$ were not statistically significant in multivariate analysis.

The mean time interval between a visual disturbance and surgery was $42.5 \pm 43.1$ days (range, 3 to 115 days). We measured corrected visual acuity and extraocular muscle limitations 1 month after endoscopic surgery. Visual acuity improved significantly in 2 of the 8 patients, and extraocular muscle restriction improved in 2 of 5 patients. The 2 patients who recovered from the visual disturbance had better preoperative visual acuity compared with that of the other patients (Table 3).

\section{DISCUSSION}

We showed that a sphenoid sinus FB occurred relatively frequently $(11 \%)$ in the paranasal sinus, and that orbital complications occurred in $17 \%$ of patients with a sphenoid sinus FB. Orbital complications were associated with old age, a bony defect in the sphenoid sinus detected on $\mathrm{CT}$, and the presence of DM.

The incidence of sphenoid sinus FB is $11 \%$ of all paranasal sinus FB cases and it comprises $2 \%$ of patients who underwent endoscopic sinus surgery for chronic rhinosinusitis in our hospital during the same period (data not shown). These incidences are similar with those of previous reports. The incidence of a sphenoid sinus FB is $13 \%$ to $25 \%$ of all paranasal sinus FB and comprises $3 \%$ of all sinus infection cases [8-10]. Friedman et al. [11] found that fungal sinusitis, of which most cases were characterized by a FB, accounted for $31 \%$ of inflammatory sphenoid diseases. A sphenoid sinus FB is often underdiagnosed because the symptoms are nonspecific, and the final diagnosis can only be made after an operation [12]. Headache and other nonspecific nasal sinus signs lasting for an extended period are the best indicators of a sphenoid sinus FB. Newly developed imaging techniques and endoscopy have made diagnosis of a FB disease easy but the incidence is increasing rapidly.

FB affects older women more frequently, with a male to female ratio of 1:2 [2,9,13]. The age distribution of our study ( $57 \%,>60$ years and $76 \%,>50$ years; mean age, 59.7 years) and the female predominance $(76 \%)$ were similar to those described in a previous study [12]. The precise mechanism for the predilection to elderly women has not been revealed.

A possible association between an immunocompromised condition and sphenoid sinus FB has been suggested previously. Toussain et al. [14] reported that patients with various immunocompromised conditions, including uncontrolled DM, neutropenia, and a previous transplant more frequently have involved deep sinus (ethmo-sphenoidal) involvement. However, none of our patients had neutropenia or were involved with a transplant. DM coexists in $23 \%$ of patients with a sphenoid sinus FB, which is similar with the frequency in the healthy Korean population $>65$ years of age $(27 \%)$.

$\mathrm{CT}$ is frequently used to detect sphenoid disease, including a FB (opacity and central microcalcification). Bony sclerosis or thickening of the bony wall was found in $64 \%$ of enrolled patients and may have originated from chronic inflammation caused by the FB. Bony wall defects were found in $40 \%$ of our patients. Lysis of the sphenoid sinus bony wall can be caused by an invasive fungal infection, but tissue invasion by fungal hyphae was absent in all of our cases. Some authors have reported these findings and considered them part of destructive non-invasive sinusitis [15].

Visual disturbances are another symptom frequently detected 
in patients with a sphenoid sinus $\mathrm{FB}$, and 8 patients $(17 \%)$ in our study presented with visual disturbances. The frequency of visual disturbance is $24 \%$ to $50 \%$ of sphenoid FB cases in previous studies $[8,16,17]$. Thus, it is important to investigate factors associated with a visual disturbance. We showed that old age, DM, and the presence of a bony wall defect in the sphenoid sinus were associated with visual disturbance. This is the first study that has investigated factors associated with sphenoid sinus FB with visual disturbance. Although DM did not co-occur frequently in patients with a sphenoid FB in this study, it was significantly associated with the occurrence of visual disturbance. Several case series have reported FB in paranasal sinuses complicated by immunocompromised patients $[18,19]$. Toussain et al. [14] reported that complicated FB in the paranasal sinuses occurs more frequently in patient with immunocompromising host factors. Lysis of the sphenoid sinus bony wall is also associated with a visual disturbance. The mechanisms of bony wall lysis is unknown, but it is regarded as a consequence of chronic inflammation induced by fungal growth [20]. Thus, older patients with DM or bony wall lysis should be considered for early removal of a sphenoid sinus FB.

It is very difficult to distinguish between invasive fungal sinusitis and sphenoid sinus FB when the disease is accompanied by a visual disturbance because a visual disturbance can be a symptom of invasive fungal sinusitis [21]. However, vision and eye movements can also be disturbed by neuritis, compression, or ischemic infarctions resulting from thrombophlebitis of the optic nerves in isolated cases of sphenoid sinus disease. The occurrence of a visual disturbance, despite the absence of mucosal invasion on a pathological examination, can be explained by these hypotheses. Diplopia, due to involvement of the sixth cranial nerve, is observed in $40 \%$ of isolated sphenoid sinus disease cases [22]. In our study, $17 \%$ of biopsies showed that patients with a sphenoid sinus FB had a visual disturbance.

A few reports are available on treatment outcomes of patients with sphenoid sinus disease with visual disturbance. Poor treatment outcomes have been reported for visual recovery, when a patient has a visual disturbance in addition to a sphenoid sinus FB [22]. Patients who have a visual disturbance have a poor visual function prognosis $[22,23]$. Regardless of the success of sphenoidotomy to remove the FB, 2 of 3 patients with partial visual loss recovered from a visual disturbance in our study, but patients with complete or near-complete visual loss did not show improved visual functioning. Because of this low recovery rate, a sphenoid sinus FB may be considered an emergent condition. Thus, surgical removal is recommended if a sphenoid sinus FB is accompanied by a visual disturbance [24]. Furthermore, early surgical removal should be considered as an option for a sphenoid sinus FB even in a patient without visual dysfunction.

In this study, we tried to determine factors significantly associated with visual disturbances in patients with a sphenoid sinus FB. Patients with a sphenoid sinus bony wall defect had higher rates of visual disturbance occurrence. Further study will be required to verify the age and DM as the associated factor. Although we could not elucidate factors significantly associated with recovery from a visual disturbance due to the small case number, visual improvement was observed only in patients with partial visual loss rather than those with complete or near-complete visual loss. If patients present with sphenoid sinus wall defect, early surgical intervention is necessary to prevent the visual disturbance from causing optic neuropathy.

\section{CONFLICT OF INTEREST}

No potential conflict of interest relevant to this article was reported.

\section{REFERENCES}

1. deShazo RD, Chapin K, Swain RE. Fungal sinusitis. N Engl J Med. 1997 Jul;337(4):254-9.

2. Park GY, Kim HY, Min JY, Dhong HJ, Chung SK. Endodontic treatment: a significant risk factor for the development of maxillary fungal ball. Clin Exp Otorhinolaryngol. 2010 Sep;3(3):136-40.

3. Kwon MO, Kim KS. Headache induced by isolated sphenoid fungal sinusitis: sinus headache? J Headache Pain. 2009 Dec;10(6):473-6.

4. Karkas A, Rtail R, Reyt E, Timi N, Righini CA. Sphenoid sinus fungus ball. Eur Arch Otorhinolaryngol. 2013 Mar;270(3):893-8.

5. Lawson W, Reino AJ. Isolated sphenoid sinus disease: an analysis of 132 cases. Laryngoscope. 1997 Dec;107(12 Pt 1):1590-5.

6. DeLano MC, Fun FY, Zinreich SJ. Relationship of the optic nerve to the posterior paranasal sinuses: a CT anatomic study. AJNR Am J Neuroradiol. 1996 Apr;17(4):669-75.

7. Heskova G, Mellova Y, Holomanova A, Vybohova D, Kunertova L, Marcekova M, et al. Assessment of the relation of the optic nerve to the posterior ethmoid and sphenoid sinuses by computed tomography. Biomed Pap Med Fac Univ Palacky Olomouc Czech Repub. 2009 Jun;153(2):149-52.

8. Lew D, Southwick FS, Montgomery WW, Weber AL, Baker AS. Sphenoid sinusitis: a review of 30 cases. N Engl J Med. 1983 Nov; 309(19):1149-54.

9. Pagella F, Pusateri A, Matti E, Giourgos G, Cavanna C, De Bernardi F, et al. Sphenoid sinus fungus ball: our experience. Am J Rhinol Allergy. 2011 Jul-Aug;25(4):276-80.

10. Pagella F, Matti E, De Bernardi F, Semino L, Cavanna C, Marone P, et al. Paranasal sinus fungus ball: diagnosis and management. Mycoses. 2007 Nov;50(6):451-6.

11. Friedman A, Batra PS, Fakhri S, Citardi MJ, Lanza DC. Isolated sphenoid sinus disease: etiology and management. Otolaryngol Head Neck Surg. 2005 Oct;133(4):544-50.

12. Klossek JM, Peloquin L, Fourcroy PJ, Ferrie JC, Fontanel JP. Aspergillomas of the sphenoid sinus: a series of 10 cases treated by endoscopic sinus surgery. Rhinology. 1996 Sep;34(3):179-83.

13. Socher JA, Cassano M, Filheiro CA, Cassano P, Felippu A. Diagnosis and treatment of isolated sphenoid sinus disease: a review of 109 cases. Acta Otolaryngol. 2008 Sep;128(9):1004-10.

14. Toussain G, Botterel F, Alsamad IA, Zerah-Lancner F, Pruliere-Escabasse V, Coste A, et al. Sinus fungal balls: characteristics and management in patients with host factors for invasive infection. Rhinolo- 
gy. 2012 Sep;50(3):269-76.

15. Grosjean P,Weber R. Fungus balls of the paranasal sinuses: a review. Eur Arch Otorhinolaryngol. 2007 May;264(5):461-70.

16. Gilain L, Aidan D, Coste A, Peynegre R. Functional endoscopic sinus surgery for isolated sphenoid sinus disease. Head Neck. 1994 SepOct;16(5):433-7.

17. Postma GN, Chole RA, Nemzek WR. Reversible blindness secondary to acute sphenoid sinusitis. Otolaryngol Head Neck Surg. 1995 Jun;112(6):742-6.

18. Ferguson BJ. Fungus balls of the paranasal sinuses. Otolaryngol Clin North Am. 2000 Apr;33(2):389-98.

19. Rowe-Jones JM, Moore-Gillon V. Destructive noninvasive paranasal sinus aspergillosis: component of a spectrum of disease. J Otolaryngol. 1994 Apr;23(2):92-6.
20. Klossek JM, Serrano E, Peloquin L, Percodani J, Fontanel JP, Pessey JJ. Functional endoscopic sinus surgery and 109 mycetomas of paranasal sinuses. Laryngoscope. 1997 Jan;107(1):112-7.

21. Fisher BD, Armstrong D, Yu B, Gold JW. Invasive aspergillosis: progress in early diagnosis and treatment. Am J Med. 1981 Oct;71(4): 571-7.

22. Lee LA, Huang CC, LeeTJ. Prolonged visual disturbance secondary to isolated sphenoid sinus disease. Laryngoscope. 2004 Jun;114(6): 986-90.

23. Patt BS, Manning SC. Blindness resulting from orbital complications of sinusitis. Otolaryngol Head Neck Surg. 1991 Jun;104(6):789-95.

24. $\mathrm{Hu} \mathrm{L}$, Wang $\mathrm{D}, \mathrm{Yu} \mathrm{H}$. Isolated sphenoid fungal sinusitis and vision loss: the case for early intervention. J Laryngol Otol. 2009 Feb;123 (2):e8. 